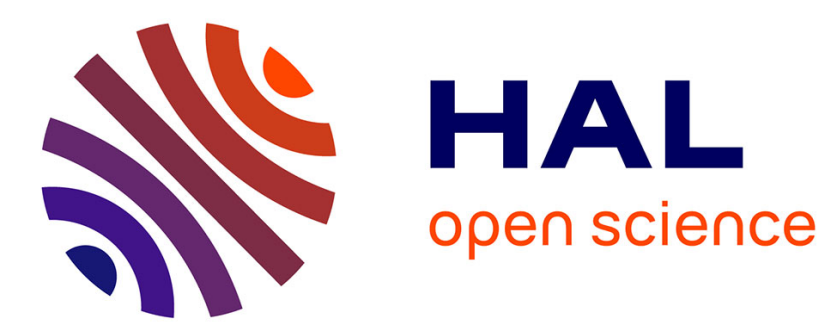

\title{
Fixed-distance multipoint formulas for the scattering amplitude from phaseless measurements
}

Roman Novikov, Vladimir Sivkin

\section{To cite this version:}

Roman Novikov, Vladimir Sivkin. Fixed-distance multipoint formulas for the scattering amplitude from phaseless measurements. Inverse Problems, 2022, 38 (2), pp.025012. 10.1088/1361$6420 / \mathrm{ac} 44 \mathrm{db}$. hal-03263803v2

\section{HAL Id: hal-03263803 \\ https://hal.science/hal-03263803v2}

Submitted on 9 Oct 2021

HAL is a multi-disciplinary open access archive for the deposit and dissemination of scientific research documents, whether they are published or not. The documents may come from teaching and research institutions in France or abroad, or from public or private research centers.
L'archive ouverte pluridisciplinaire HAL, est destinée au dépôt et à la diffusion de documents scientifiques de niveau recherche, publiés ou non, émanant des établissements d'enseignement et de recherche français ou étrangers, des laboratoires publics ou privés. 


\title{
Fixed-distance multipoint formulas for the scattering amplitude from phaseless measurements
}

\author{
R.G. Novikov ${ }^{1,2}$, V.N. Sivkin ${ }^{1,3}$ \\ ${ }^{1}$ CMAP, CNRS, Ecole Polytechnique, Institut Polytechnique de Paris, 91128 Palaiseau, France \\ 2 IEPT RAS, 117997 Moscow, Russia \\ ${ }^{3}$ Lomonosov MSU, Moscow, 119991, Russia \\ Email: novikov@cmap.polytechnique.fr, sivkin96@yandex.ru,
}

\begin{abstract}
We give new formulas for finding the complex (phased) scattering amplitude at fixed frequency and angles from absolute values of the scattering wave function at several points $x_{1}, \ldots, x_{m}$. In dimension $d \geq 2$, for $m>2$, we significantly improve previous results in the following two respects. First, geometrical constraints on the points needed in previous results are significantly simplified. Essentially, the measurement points $x_{j}$ are assumed to be on a ray from the origin with fixed distance $\tau=\left|x_{j+1}-x_{j}\right|$, and high order convergence (linearly related to $m$ ) is achieved as the points move to infinity with fixed $\tau$. Second, our new asymptotic reconstruction formulas are significantly simpler than previous ones. In particular, we continue studies going back to [Novikov, Bull. Sci. Math. 139(8), 923-936, 2015].
\end{abstract}

Keywords: Schrödinger equation, Helmholtz equation, Monochromatic scattering data, Phase retrieval, Phaseless inverse scattering

AMS subject classification: 35J10, 35P25, 35R30, 81U40

\section{Introduction}

We consider monochromatic scattering modelled using the equation

$$
-\Delta \psi+v(x) \psi=E \psi, x \in \mathbb{R}^{d}, d \geq 1, E>0,
$$

where

$$
\begin{aligned}
& v \in L^{\infty}(D), \quad v \equiv 0 \text { on } \mathbb{R}^{d} \backslash D, \\
& D \text { is an open bounded domain in } \mathbb{R}^{d} .
\end{aligned}
$$

We assume that $v$ is complex-valued. The regularity assumption that $v \in L^{\infty}(D)$ is just for simplicity and can be relaxed; see Remark 2.1. The main point is that for equation (1), under such an assumption, we can consider the scattering solutions $\psi^{+}$specified via Sommerfeld type radiation condition like (3).

Equation (1) arises in quantum mechanics as the Schrödinger equation at fixed energy and in acoustics and electrodynamics as the Helmholtz equation at fixed frequency. Under assumption (2), the coefficient $v$ describes a scatterer contained in $D$. The number $E$ is related to the timeharmonic frequency and corresponds to the energy in the framework of the Schrödinger equation. In addition, $v$ may depend on $E$, at least, in acoustics and electrodynamics. See, for example, [5], [7], [9], [13].

For equation (1) we consider the solutions $\psi^{+}(x, k), k \in \mathbb{R}^{d}, k^{2}=E$, specified by the following asymptotic as $|x| \rightarrow \infty$ :

$$
\psi^{+}(x, k)=e^{i k x}+\frac{e^{i|k||x|}}{|x|^{(d-1) / 2}} f_{1}\left(k,|k| \frac{x}{|x|}\right)+\mathcal{O}\left(\frac{1}{|x|^{(d+1) / 2}}\right),
$$

for some a priori unknown $f_{1}$. The solutions $\psi^{+}=\psi^{+}(x, k)$ are the scattering solutions, or scattering wave functions, for equation (1). These solutions describe scattering of the incident plan waves described by $e^{i k x}$ on the scatterer described by $v$. In particular, the second term on 
the right-hand side of (3) describes the leading scattered spherical waves. The coefficient $f_{1}$ arising in (3) is a function defined on

$$
\mathcal{M}_{E}=\left\{k, l \in \mathbb{R}^{d}: k^{2}=l^{2}=E\right\}=\mathbb{S}_{\sqrt{E}}^{d-1} \times \mathbb{S}_{\sqrt{E}}^{d-1},
$$

where $l=|k| \frac{x}{|x|}, \mathbb{S}_{r}^{d-1}$ is the sphere of radius $r$ centered at the origin in $\mathbb{R}^{d}$.

The function $f_{1}$ is the scattering amplitude, or far field pattern, for equation (1).

In order to study $\psi^{+}$and $f_{1}$ one can use, in particular, the Lippmann-Schwinger integral equation (16) for $\psi^{+}$and formulas (20)-(22) for $f_{1}$; see Subsection 2.1.

We recall that in quantum mechanics the complex values of the functions $\psi^{+}$and $f_{1}$ have no direct physical sense, whereas the phaseless values of $\left|\psi^{+}\right|^{2}$ and $\left|f_{1}\right|^{2}$ have probabilistic interpretation (according to the Born's rule) and can be directly measured. See [6] and, for example, [12], for details. In turn, in acoustics or electrodynamics the complex values of $\psi^{+}$and $f_{1}$ can be directly measured, at least, in principle. However, in electro-magnetic wave propagation at very high frequencies (as for $X$-rays and lasers) only phaseless values of $\left|\psi^{+}\right|^{2}$ and $\left|f_{1}\right|^{2}$ can be measured in practice by modern technical devices; see, e.g., [17] and references therein.

For equation (1) under assumptions (2), we consider, in particular, the following problems:

Problem 1.1. Reconstruct potential $v$ from its scattering amplitude $f_{1}$.

Problem 1.2. Reconstruct potential $v$ from its phaseless scattering data $\left|\psi^{+}\right|^{2}$ appropriately given outside of $D$.

Problem 1.3. Find $f_{1}$ from $\left|\psi^{+}\right|^{2}$ appropriately given outside of $D$.

A recent survey on these problems is given in [30]. Actually, in the present work we continue studies of [11], [26]-[31], [33] on Problem 1.3. These studies on Problem 1.3 and results on Problem 1.1 admit straightforward applications to Problem 1.2. For other possible approaches to Problem 1.2, see, for example, [40], [41], [22], [10], [16], [23], [24], [19], [35].

In particular, in the present work we give, for fixed $(k, l) \in \mathcal{M}_{E}, l \neq k$, for $d \geq 2$,

$$
\begin{aligned}
& \text { formulas for finding } f_{1}(k, l) \text { up to } \mathcal{O}\left(s^{-n}\right) \text { as } s \rightarrow+\infty \text {, } \\
& \text { from }\left|\psi^{+}(x, k)\right|^{2} \text { given at } m \text { points } x=x_{1}(s), \ldots, x_{m}(s) \text {, }
\end{aligned}
$$

where $m$ depends linearly on $n$,

$$
\begin{aligned}
& x_{j}(s)=\left(s+\tau_{j}\right) \hat{l}, j=1, \ldots, m, \hat{l}=l /|l|, \\
& s>0, \tau_{1}=0, \tau_{j_{1}}<\tau_{j_{2}}, j_{1}<j_{2} .
\end{aligned}
$$

These formulas are explicit and are presented in detail below in Introduction and in Section 3, where our precise assumptions on $m=m(n)$ and on $\tau_{1}, \ldots, \tau_{m}$ are specified.

One can see that in formulas $(5),(6)$ the measurement points $x_{j}=x_{j}(s)$ are on the ray starting at the origin in direction $\hat{l}$, where $s$ is the distance between the origin and the set of these points, and the distances $\tau_{j+1}-\tau_{j}=\left|x_{j+1}-x_{j}\right|$ are fixed. In addition, the reconstruction formulas mentioned in (5) are asymptotic, where $n$ can be considered as their convergence rate in terms of $\mathcal{O}\left(s^{-n}\right)$ as $s \rightarrow+\infty$, that is when the points $x_{j}=x_{j}(s)$ move to infinity.

Note that, to our knowledge, for the first time formulas (5), (6) were realized in [26], [28] for $n=1, m=2, d \geq 3$ and for $n=1 / 2, m=2, d=2$, and in [29] for $n=1, m=2, d=2$. In addition, for $m=3, d=1$, an exact (not asymptotic !) analog of formulas (5), (6) was given in [27]. In the present work, for the first time we realize formulas (5), (6) for $n>1, d \geq 2$. In this respect we proceed from studies recently developed in [29] and [31]; more comments are given below in Introduction and in Section 2.

Let

$$
a(x, k)=|x|^{(d-1) / 2}\left(\left|\psi^{+}(x, k)\right|^{2}-1\right),
$$

where $x, k \in \mathbb{R}^{d} \backslash\{0\}$. 
Assume in (5), (6) that $d=3, m=2 n, n \in \mathbb{N}=\{1,2,3, \ldots\}$, and that

$$
\begin{aligned}
& \tau_{j}=\left\{\begin{array}{l}
(j-1) \tau, j=1, \ldots, n, \\
\sigma+(j-1-n) \tau, j=n+1, \ldots, 2 n,
\end{array}\right. \\
& \tau=\tau(k, l)=\frac{2 \pi}{\kappa}, 0<\sigma \neq 0\left(\bmod \frac{\pi}{\kappa}\right), \kappa=\kappa(k, l)=|k|-k \hat{l} .
\end{aligned}
$$

Then our formulas (5), (6) are as follows:

$$
\begin{aligned}
& f_{1}(k, l)=\frac{e^{-i(s+\sigma) \kappa} a_{1}(s)-e^{-i s \kappa} a_{2}(s)+\mathcal{O}\left(s^{-n}\right)}{-2 i \sin (\sigma \kappa)}, s \rightarrow+\infty, \\
& a_{1}(s)=a_{1}(k, l, s)=\sum_{j=1}^{n} \frac{(-1)^{n-j}\left(s+\tau_{j}\right)^{n-1} a\left(x_{j}(s), k\right)}{(j-1) !(n-j) ! \tau^{n-1}}, \\
& a_{2}(s)=a_{2}(k, l, s)=\sum_{j=n+1}^{2 n} \frac{(-1)^{j}\left(s+\tau_{j}\right)^{n-1} a\left(x_{j}(s), k\right)}{(j-1-n) !(2 n-j) ! \tau^{n-1}}
\end{aligned}
$$

where $(k, l) \in \mathcal{M}_{E}, l \neq k, d=3, a(x, k)$ is defined by (7), $x_{j}(s)$ are defined in (6), (8), (9), $\tau, \tau_{j}, \sigma, \kappa$ are the numbers of (8), (9).

Formulas (10)-(12) are new for $n \geq 2$; for $n=1$ these formulas were given in [26], [28].

Somewhat more general version of formulas (10)-(12) is given as Theorem 3.1; see Subsection 3.1.

One can see that formulas (10)-(12) and formulas of Theorem 3.1 are completely explicit ! However, a possible practical inconvenience of these formulas is that the differences between the measurement points $x_{j}(s), j=1, \ldots, n$, or $x_{j}(s), j=n+1, \ldots, 2 n$, in (10)-(12) (and between the related points in Theorem 3.1) are multiple to $\tau=\tau(k, l)=2 \pi / \kappa$, where $\kappa=\kappa(k, l)$ is defined in (9). The inconvenience is that this $\tau$ depend on $k, l$. Besides, these formulas are valid for $d=3$ but are not valid for $d=2$ (because of slightly different structure of the asymptotic expansions (3), (19) for $\psi^{+}$for $d=3$ and for $d=2$ ). Therefore, in the present work we also give the following further results without the aforementioned multiplicity condition for the differences between $x_{j}(s)$, for $d \geq 2$.

We give an explicit version of formulas (5), (6) for the case of the linearised Problem 1.3 (near $v=0$ ) for $d \geq 2, m=2 n, n \in \mathbb{N}$, where

$$
\tau_{j}=(j-1) \tau, j=1, \ldots, 2 n, \tau>0, \tau \neq 0\left(\bmod \frac{\pi}{\kappa}\right),
$$

$\kappa=\kappa(k, l)$ in defined in $(9)$; see Theorem 3.2 of Subsection 3.2.

We give explicit versions of formulas (5), (6) for the general non-linearised case for $d=3, m=$ $3 n-1$, and for $d=2, m=3 n$, where

$$
\tau_{j}=(j-1) \tau, j=1, \ldots, m, \tau>0, \tau \neq 0\left(\bmod \frac{\pi}{\kappa}\right),
$$

$\kappa=\kappa(k, l)$ in defined in (9); see Proposition 3.1 of Subsection 3.3 and Proposition 3.2 of Subsection 3.4. For the general non-linearized case, finding such formulas for $m=2 n$ is an open question for $n>1$, for $d=3$ or $d=2$, under assumption (14). We recall that for $n=1, d=3$, this question was solved in [26], [28]; whereas for $n=1, d=2$, this question was solved in Section 9 of [29].

Note that $\tau$ in (13), (14) is fixed and independent of $k, l$ (except the property that $\tau \neq$ $0(\bmod \pi / \kappa))$ in contrast with $\tau$ in $(9)$.

The results of the present work are obtained proceeding from methods developed in [26], [29], [31]. In particular, for fixed $(k, l) \in \mathcal{M}_{E}, l \neq k$, for $d=3$ or $d=2$, the work [29] gives a version of formulas (5), where

$$
\begin{aligned}
& x_{j}(s)=r_{j}(s) \hat{l}, j=1, \ldots, 2 n, \hat{l}=l /|l|, \\
& r_{2 i-1}(s)=\lambda_{i} s, r_{2 i}(s)=\lambda_{i} s+\tau, i=1, \ldots, n, \\
& \lambda_{1}=1, \lambda_{i_{1}}<\lambda_{i_{2}} \text { for } i_{1}<i_{2}, \tau>0 .
\end{aligned}
$$


Formulas (5), (15) realized in [29] are recurrent in $n$. For $n=1, d=3$, these formulas were given in [26].

Advantages of formulas (5), (6) (realized in the present work) in comparison with formulas (5), (15) (realized in [29]) can be summarized as follows:

(a) The geometry of $x_{j}(s)$ in (5), (6) is essentially simpler in the sense that the distances between all these points are fixed and are independent of $s \rightarrow+\infty$.

(b) Formulas (5), (6) (realized as formulas (10)-(12), (53)-(55), (64)-(66), (72)-(75), (83)(85)) are drastically more explicit for large $n$.

Note that the results of the present work essentially use the technique of the recent work [31], where [31] gives explicit asymptotic multipoint formulas for finding $f$ from $\psi^{+}$.

Note also that explicit estimates on the reminder $\mathcal{O}\left(s^{-n}\right)$ in our formulas (5), (6) can be given proceeding from methods developed in [31], [33].

Numerical aspects of formulas of [26], [28], [29], [33] and of the present article with their applications to Problem 1.2 will be addressed in further works.

In addition to Problem 1.3 and Problem 1.2, there are also other possible formulations of phase retrieval and phaseless inverse scattering problems for equation (1) and for other equations of wave propagations. In connection with such other formulations and related results, see, for example, [8], [13], [15], [17], [18], [20], [28], [30], [34], [36], [37], [38], [42] and references therein. Note that formulas of [26], [28], [29] and the present work can be also used for Problems 1.3 and 1.2 when coefficient $v$ in equation (1) is replaced, e.g., by an impenetrable obstacle (see, e.g., [9] for definition of impenetrable obstacles).

The further structure of the present article is as follows. In Section 2 we recall, in particular, some results on direct scattering for equation (1) under assumptions (2) and some formulas of [29] and [31]. The results of the present work on Problem 1.2, consisting in realizations of formulas (5), (6), are given in Section 3. These results are proved in Sections 4, 5, and 6. In Section 7, for completeness of presentation, we give a sketch of proof of formulas (23)-(25) for the higher scattering amplitudes $f_{j}, j \geq 2$.

\section{Preliminaries}

\subsection{Asymptotics of the scattering solutions}

We recall that the scattering solutions $\psi^{+}$satisfy the following Lippmann-Schwinger integral equation:

$$
\begin{aligned}
& \psi^{+}(x, k)=e^{i k x}+\int_{D} G^{+}(x-y, k) v(y) \psi^{+}(y, k) d y, \\
& G^{+}(x, k):=-(2 \pi)^{-d} \int_{\mathbb{R}^{d}} \frac{e^{i \xi x} d \xi}{\xi^{2}-k^{2}-i \cdot 0}=G_{0}^{+}(|x|,|k|),
\end{aligned}
$$

where $x, k \in \mathbb{R}^{d}, k^{2}=E$; see, for example, [5], [9], [30].

Note also that

$$
\begin{aligned}
& G^{+}(x, k)=\frac{e^{i|k||x|}}{2 i|k|}, d=1, \\
& G^{+}(x, k)=-\frac{i}{4} H_{0}^{1}(|x||k|), d=2, G^{+}(x, k)=-\frac{e^{i|k||x|}}{4 \pi|x|}, d=3,
\end{aligned}
$$

where $H_{0}^{1}$ is the Hankel function of the first type.

Actually, in the present work, in addition to (2), we assume that, for fixed $E>0$,

$$
\text { equation (16) is uniquely solvable for } \psi^{+}(\cdot, k) \in L^{\infty}(D) \text {. }
$$


In addition, if $v$ satisfies (2) and is real-valued, then (18) is fulfilled automatically.

Remark 2.1. The regularity assumption that $v \in L^{\infty}(D)$ can be essentially relaxed in (2) (although this assumption is used often in the literature). In the results of Section 3 on Problem 1.3 in place of $(2),(18)$, it is sufficient to assume that $v$ supported in $D$ is such that:

equation (1) for fixed $E>0$ has an unique solution $\psi^{+}(\cdot, k)$ specified via Sommerfeld type radiation condition like (3) for each $k \in \mathbb{R}^{d}, k^{2}=E$, in Subsections 3.1, 3.3, 3.4;

and in addition $\psi^{+}(x, k)-e^{i k x}$ is small on $\partial D$ if $v$ is small, where $\partial D$ is regular, in Subsection 3.2.

Proceeding, for example, from (16) one can show that the scattering solutions $\psi^{+}$have the following Atkinson-type expansion:

$$
\psi^{+}(x, k)=e^{i k x}+\frac{e^{i|k||x|}}{|x|^{(d-1) / 2}}\left(\sum_{j=1}^{N} \frac{f_{j}\left(k,|k| \frac{x}{|x|}\right)}{|x|^{j-1}}+\mathcal{O}\left(\frac{1}{|x|^{N}}\right)\right),|x| \rightarrow+\infty, N \in \mathbb{N},
$$

where the coefficients $f_{j}$ arising in (19) are functions defined on $\mathcal{M}_{E}$; see [3], [39], [25], [29], [31].

Formula (19) for $N=1$ reduces to (3). We say that the functions $f_{j}$ for $j \geq 2$ are the higher scattering amplitudes for equation (1).

It is well-known that

$$
\begin{aligned}
& f_{1}(k, l)=c(d,|k|) f(k, l), \\
& c(d,|k|)=-\pi i(-2 \pi i)^{(d-1) / 2}|k|^{(d-3) / 2}, \text { for } \sqrt{-2 \pi i}=\sqrt{2 \pi} e^{-i \pi / 4}, \\
& f(k, l)=(2 \pi)^{-d} \int_{D} e^{-i l y} v(y) \psi^{+}(y, k) d y,
\end{aligned}
$$

where $(k, l) \in \mathcal{M}_{E}$; see, for example, [30].

It is also well-known that $f_{j} \equiv 0$ for $j \geq 2, d=1$, under assumptions (2), (18).

Besides, in the present work we also use the following formulas, for $d \geq 2$ :

$$
\begin{aligned}
& f_{j}(k, l)=(2 \pi)^{-d} c(d,|k|) \int_{D} \varphi_{j}(y, l) v(y) \psi^{+}(y, k) d y, j \geq 1, \\
& \varphi_{1}(y, l)=e^{-i l y}, \\
& \varphi_{j}(y, l)=\frac{\left(d-3 / 4-d^{2} / 4+(j-1)(j-2)\right) \varphi_{j-1}(y, l)+\Delta_{S} \varphi_{j-1}(y, l)}{2 i|l|(j-1)}, j \geq 2,
\end{aligned}
$$

where $\Delta_{S}$ is the Beltrami-Laplace operator on the unit sphere $\mathbb{S}^{d-1}$, acting with respect to $\hat{l}=l /|l|$. Formulas (23)-(24) follow from (20)-(22) and the following Barrar-Kay-Wilcox type recursion formulas:

$$
f_{j}(y, l)=\frac{\left(d-3 / 4-d^{2} / 4+(j-1)(j-2)\right) f_{j-1}(y, l)+\Delta_{S} f_{j-1}(y, l)}{2 i|l|(j-1)}, j \geq 2,
$$

see [39] in connection with (26) for $d=3$.

For completeness of the presentation, a sketch of proof of formulas (23)-(25), for $j \geq 2$, is given in Section 7. Note that the precise form of the recurrent relations (25) is not essential for the main results of the present work.

\subsection{Asymptotic formulas for $a(x, k)$}

Consider the function $a=a(x, k)$ defined by (7). Let $\kappa=\kappa\left(k,|k| \frac{x}{|x|}\right)$ be defined as in (9). Let

$$
f_{j}=f_{j}\left(k,|k| \frac{x}{|x|}\right)
$$


where $f_{j}$ are the functions arising in (19), $j \in \mathbb{N}, x, k \in \mathbb{R}^{d} \backslash\{0\}$. Then $a(x, k)$ can be presented as follows (see [29] for $d=3$ or $d=2$ ):

$$
\begin{aligned}
& a(x, k)=a_{N}(x, k)+\delta_{N} a(x, k), \\
& a_{N}(x, k)=a_{N}^{1}(x, k)+a_{N}^{2}(x, k), \\
& a_{N}^{1}(x, k)=\sum_{j=1}^{N} \frac{e^{i|x| \kappa} f_{j}}{|x|^{j-1}}+\sum_{j=1}^{N} \frac{e^{-i|x| \kappa} \bar{f}_{j}}{|x|^{j-1}}, \\
& a_{N}^{2}=\sum_{j=1}^{N-[(d-1) / 2]} \frac{h_{j}}{|x|^{j-1+(d-1) / 2}}, h_{j}=\sum_{\alpha=1}^{j} f_{\alpha} \bar{f}_{j-\alpha+1}, \\
& \delta_{N} a(x, k)=\mathcal{O}\left(|x|^{-N}\right), \text { as }|x| \rightarrow+\infty,
\end{aligned}
$$

where $x \in \mathbb{R}^{d} \backslash\{0\}, k \in \mathbb{R}^{d}, k^{2}=E>0, d \geq 2,[\cdot]$ stands for the integer part.

Note that formulas (7), (19) imply formulas (28)-(32) as follows:

$$
\begin{aligned}
& a(x, k)=a_{N}^{1}(x, k)+\mathcal{O}\left(|x|^{-N}\right)+\frac{1}{|x|^{(d-1) / 2}}\left(\sum_{j_{1}=1}^{N} \frac{f_{j_{1}}}{|x|^{j_{1}-1}}+\mathcal{O}\left(|x|^{-N}\right)\right)\left(\sum_{j_{2}=1}^{N} \frac{\bar{f}_{j_{2}}}{|x|^{j_{2}-1}}+\mathcal{O}\left(|x|^{-N}\right)\right)= \\
& =a_{N}^{1}(x, k)+\mathcal{O}\left(|x|^{-N}\right)+\frac{1}{|x|^{(d-1) / 2}}\left(\sum_{j_{1}, j_{2}=1}^{N} \frac{f_{j_{1}} \bar{f}_{j_{2}}}{|x|^{j_{1}-1}|x|^{\mid j_{2}-1}}+\mathcal{O}\left(|x|^{-N}\right)\right) \stackrel{j=j_{1}+j_{2}-1}{=} \\
& =a_{N}^{1}(x, k)+\mathcal{O}\left(|x|^{-N}\right)+\frac{1}{|x|^{(d-1) / 2}}\left(\sum_{j=1}^{N} \sum_{j_{1}=1}^{j} \frac{f_{j_{1}} \bar{f}_{j-j_{1}+1}}{|x|^{j-1}}+\mathcal{O}\left(|x|^{-N}\right)\right)= \\
& =a_{N}^{1}(x, k)+\sum_{j=1}^{N-[(d-1) / 2]} \frac{h_{j}}{|x|^{j-1+(d-1) / 2}}+\mathcal{O}\left(|x|^{-N}\right)
\end{aligned}
$$

where we used the change of variables $\left(j_{1}, j_{2}\right) \rightarrow\left(j_{1}, j\right), j=j_{1}+j_{2}-1$.

Consider also the case of the Born approximation for small potentials. Suppose that potential $v$ is small, for example, in the sense of the norm $\|\cdot\|_{L^{\infty}(D)}$, for fixed $D$. Then in formulas (28)-(32) the quadratic term $a_{N}^{2}$ is negligible in comparison with the linear term $a_{N}^{1}$. Such a situation arises in many applications (see, for example, [6], [13], [16], [23], [24], [40], [41]). Thus, in the Born approximation for small potentials formula (29) reduces to the formula

$$
a_{N}(x, k) \approx a_{N}^{1}(x, k)
$$

The aforementioned smallness assumption on $v$ can be specified as

$$
\|v\|_{L^{\infty}(D)}=\mathcal{O}(\varepsilon), \text { where } \varepsilon \rightarrow 0 .
$$

Then using (16), (20)-(25), (35) one can show that

$$
\left\|f_{j}\right\|_{C\left(\mathcal{M}_{E}\right)}=\mathcal{O}(\varepsilon), \text { for each } j \in \mathbb{N} .
$$

In turn, formulas (29), (31) imply that

$$
a_{N}(x, k)-a_{N}^{1}(x, k)=|x|^{-(d-1) / 2} \mathcal{O}\left(\varepsilon^{2}\right),
$$

where $N \in \mathbb{N}$.

Formula (37) specifies (34) under assumption (35). 


\subsection{Some results of [31]}

We recall that the work [31] gives, in particular, explicit asymptotic multipoint formulas for finding $f_{1}$ from $\psi^{+}$for $d \geq 2$. This work proceeds from formula (19) and considers, in particular, the functions $z=z(s), s \in[r,+\infty), r>0$, of the form

$$
z(s)=\sum_{j=1}^{N} \frac{f_{j}}{s^{j-1}}+\mathcal{O}\left(s^{-N}\right), \text { as } s \rightarrow+\infty,
$$

where $f_{j}, j=1, \ldots, n$, are the complex numbers.

Functions of the form (38) arise in the second term on the right-hand side of (19), where $s=|x|$. Functions of the form (38) also arise in the framework of direct and inverse scattering at high energies for equation (1) with smooth $v$; see [25] and [32].

For functions $z$ satisfying (38) the work [31] considers, in particular, the problem of finding $f_{1}$ from $z(s)$ given at $n$ points $s_{j} \in[r,+\infty), j=1, \ldots, n$, of the form

$$
\begin{aligned}
& s_{j}=s_{j}(s)=s+\tau_{j}, j=1, \ldots, n, \\
& s>r, \tau_{1}=0, \tau_{j+1}>\tau_{j}, j=1, \ldots, n-1, \\
& \vec{\tau}=\left(\tau_{1}, \ldots, \tau_{n}\right) .
\end{aligned}
$$

Suppose that $N \geq 2 n-1$. Then the following formulas of [31] hold:

$$
f_{1}=\sum_{j=1}^{n} y_{j}(s, \vec{\tau}) z\left(s+\tau_{j}\right)+\mathcal{O}\left(s^{-n}\right), \text { as } s \rightarrow+\infty,
$$

where $y_{j}(s, \vec{\tau})$ are defined by

$$
\sum_{j=1}^{n} \frac{y_{j}(s, \vec{\tau})}{\left(s+\tau_{j}\right)^{i-1}}= \begin{cases}1, & \text { for } i=1 \\ 0, & \text { for } i=2, \ldots, n\end{cases}
$$

in addition:

$$
\begin{aligned}
& y_{j}(s, \vec{\tau})=\frac{(-1)^{n-j}\left(s+\tau_{j}\right)^{n-1}}{\alpha_{j}(\vec{\tau}) \beta_{n, j}(\vec{\tau})}, 1 \leq j \leq n, \\
& \alpha_{j}(\vec{\tau})=\prod_{k=1}^{j-1}\left(\tau_{j}-\tau_{k}\right), \beta_{n, j}(\vec{\tau})=\prod_{k=j+1}^{n}\left(\tau_{k}-\tau_{j}\right), \\
& \sum_{j=1}^{n} \frac{y_{j}(s, \vec{\tau})}{\left(s+\tau_{j}\right)^{i-1}}=\mathcal{O}\left(s^{-n}\right) \text { as } s \rightarrow+\infty, \text { for } n<i<2 n .
\end{aligned}
$$

\section{Main results}

\subsection{Formulas for finding $f_{1}$ from $a$ at $2 n$ points for $d=3$}

For $d=3$, the function $a(x, k)$ of formulas (7), (28)-(32) at fixed $k$ and $\hat{x}=x /|x|$ can be written as

$$
\begin{aligned}
& a(s)=a(s, \hat{x}, k):=a(s \hat{x}, k), \\
& a(s)=\sum_{j=1}^{N} \frac{e^{i s \kappa} f_{j}+e^{-i s \kappa} \bar{f}_{j}+h_{j}}{s^{j-1}}+\mathcal{O}\left(s^{-N}\right), s \rightarrow+\infty, h_{1}=0, \\
& h_{j}=\sum_{k=1}^{j-1} f_{k} \bar{f}_{j-k}, j=1, \ldots, N,
\end{aligned}
$$


where $s=|x|, \kappa=|k|-k \hat{x}$, and $\kappa>0$ if $\hat{x}=x /|x| \neq \hat{k}=k /|k|$.

Proceeding from this motivation we consider arbitrary functions $a=a(s), s \in[r,+\infty), r>0$, such that formula (46) holds for some fixed $\kappa>0$ and some complex numbers $f_{j}, j=1, \ldots, N$, and $h_{j}, j=2, \ldots, N$.

We consider $2 n$ points $s_{1, j}, s_{2, j} \in[r,+\infty), j=1, \ldots, n$, of the form

$$
\begin{aligned}
& s_{1, j}=s_{1, j}(s)=s+\tau_{1, j}, j=1, \ldots, n, \\
& s_{2, j}=s_{2, j}(s)=s+\sigma+\tau_{2, j}, j=1, \ldots, n, \\
& \tau_{1, j}=\nu_{1, j} \tau, j=1, \ldots, n, \tau=\frac{2 \pi}{\kappa}, 0=\nu_{1,1}<\nu_{1,2}<\ldots<\nu_{1, n},\left\{\nu_{1, j}\right\} \in \mathbb{N}, \\
& \tau_{2, j}=\nu_{2, j} \tau, j=1, \ldots, n, \tau=\frac{2 \pi}{\kappa}, 0=\nu_{2,1}<\nu_{2,2}<\ldots<\nu_{2, n},\left\{\nu_{2, j}\right\} \in \mathbb{N}, \\
& s>r, \sigma>0, \sigma \neq 0\left(\bmod \frac{\pi}{\kappa}\right) .
\end{aligned}
$$

Theorem 3.1. Let $a=a(s)$ satisfy (46) for some $N \geq 2 n-1, n \in \mathbb{N}$. Then a $(s)$ at $2 n$ points $s_{1, j}, s_{2, j}$ of (48)-(52) approximately determines $f_{1}$ as follows:

$$
\begin{aligned}
& f_{1}=\frac{e^{-i(s+\sigma) \kappa} a_{1}(s)-e^{-i s \kappa} a_{2}(s)+\mathcal{O}\left(s^{-n}\right)}{-2 i \sin (\sigma \kappa)}, s \rightarrow+\infty, \\
& a_{1}(s):=\sum_{j=1}^{n} \frac{(-1)^{n-j}\left(s_{1, j}(s)\right)^{n-1} a\left(s_{1, j}(s)\right)}{\tau^{n-1} \prod_{k=1}^{j-1}\left(\nu_{1, j}-\nu_{1, k}\right) \Pi_{k=j+1}^{n}\left(\nu_{1, k}-\nu_{1, j}\right)}, \\
& a_{2}(s):=\sum_{j=1}^{n} \frac{(-1)^{n-j}\left(s_{2, j}(s)\right)^{n-1} a\left(s_{2, j}(s)\right)}{\tau^{n-1} \prod_{k=1}^{j-1}\left(\nu_{2, j}-\nu_{2, k}\right) \Pi_{k=j+1}^{n}\left(\nu_{2, k}-\nu_{2, j}\right)} .
\end{aligned}
$$

Remark 3.1. Suppose that $\nu_{1, j}=\nu_{2, j}=j-1, j=1, \ldots, n$. Then formulas (54), (55) reduce to the formulas

$$
\begin{aligned}
& a_{1}(s)=\sum_{j=1}^{n} \frac{(-1)^{n-j}\left(s_{1, j}(s)\right)^{n-1} a\left(s_{1, j}(s)\right)}{(j-1) !(n-j) ! \tau^{n-1}}, \\
& a_{2}(s)=\sum_{j=1}^{n} \frac{(-1)^{n-j}\left(s_{2, j}(s)\right)^{n-1} a\left(s_{2, j}(s)\right)}{(j-1) !(n-j) ! \tau^{n-1}} .
\end{aligned}
$$

Theorem 3.1 is proved in Section 4 .

Formulas (53)-(57) of Theorem 3.1 and Remark 3.1 are completely explicit ! But a possible inconvenience of these formulas is that the differences between the points $s_{1, j}, j=1, \ldots, n$, or $s_{2, j}, j=1, \ldots, n$, are multiple to $2 \pi / \kappa$. Below in Subsections 3.2, 3.3, we give approaches for relaxing this multiplicity condition.

Formulas (7), (45), (48)-(57) realize (5), (6) for $d=3$. In addition, due to formulas (7), (33), function $a(s)$ defined in (45) satisfies (46) for any odd $d \geq 3$ for some $h_{j}$, where $h_{1}=h_{2}=\ldots=$ $h_{(d-1) / 2}=0$ (and further $h_{j}$ are given by analogs of (33) for odd $d>3$ ). Therefore, formulas (48)-(57) remain valid for any odd $d \geq 3$; in fact, these formulas follow just from (46).

\subsection{Formulas for finding $f_{1}$ from $a$ at $2 n$ points for small potentials}

In the Born approximation for small potentials $v$ formula (29) reduces to formula (34).

In these framework, the function $a(x, k)$ of $(28)-(32)$ at fixed $k$ and $\hat{x}=x /|x|$, for $d \geq 2$, can be written as

$$
a(x, k) \approx a(s)
$$


where

$$
a(s)=\sum_{j=1}^{N} \frac{e^{i s \kappa} f_{j}+e^{-i s \kappa} \bar{f}_{j}}{s^{j-1}}+\mathcal{O}\left(s^{-N}\right), s \rightarrow+\infty,
$$

where $s=|x|, \kappa=|k|-k \hat{x}$, and $\kappa>0$ if $\hat{x}=x /|x| \neq \hat{k}=k /|k|$. Formulas (58), (59) follow from (28), (30), (32), (34).

Proceeding from this motivation, we consider arbitrary functions $a=a(s), s \in[r,+\infty), r>0$, such that formula (59) holds for some fixed $\kappa>0$ and some complex numbers $f_{j}, j=1, \ldots, N$.

We consider $2 n$ points $s_{j} \in[r,+\infty), j=1, \ldots, 2 n$, of the form

$$
\begin{aligned}
& s_{j}=s_{j}(s)=s+\tau_{j}, j=1, \ldots, 2 n, \\
& \tau_{j}=(j-1) \tau, j=1, \ldots, 2 n, \\
& s>r, \tau>0, \tau \neq 0\left(\bmod \frac{\pi}{\kappa}\right) .
\end{aligned}
$$

Let $\Sigma_{n, \tau}$ be the operator acting on functions $u$ on $[r,+\infty)$ and defined by the formula

$$
\Sigma_{n, \tau} u(s)=\sum_{j=1}^{n} \frac{(-1)^{n-j}\left(s_{j}(s)\right)^{n-1} u\left(s_{j}(s)\right)}{(j-1) !(n-j) ! \tau^{n-1}}, s \in[r,+\infty),
$$

where $s_{j}$ are defined according to $(60)-(62), j=1, \ldots, n$.

Theorem 3.2. Let $a=a(s)$ satisfy (59) for some $N \geq 2 n-1, n \in \mathbb{N}$. Let $\Sigma_{n, \tau}$ be defined by (63). Then $a(s)$ at $2 n$ points $s_{j}$ of (60)-(62) approximately determines $f_{1}$ as follows:

$$
\begin{aligned}
& f_{1}=C(\kappa, \tau, n)\left(\tau^{n-1}\left(e^{-2 i \tau \kappa} a_{2}(s)-a_{2}(s+\tau)\right)+\mathcal{O}\left(s^{-n}\right)\right), s \rightarrow+\infty, \\
& a_{2}(s):=\Sigma_{n, \tau} a_{1}(s), a_{1}(s):=\frac{e^{-2 i s \kappa}}{s^{n-1}} \Sigma_{n, \tau}\left(e^{i s \kappa} a\right)(s), \\
& C(\kappa, \tau, n):=\frac{(n-1) !}{\left(e^{2 i \tau \kappa}-1\right)^{n-1}\left(e^{-2 i \tau \kappa}-1\right)} .
\end{aligned}
$$

Remark 3.2. For $\Sigma_{n, \tau}$ defined by (63) and $2 n$ points $s_{j}$ of (60)-(62), we have that

$$
\begin{aligned}
& \Sigma_{n, \tau}\left(w_{2} \Sigma_{n, \tau}\left(w_{1} u\right)\right)(s)= \\
& =\sum_{1 \leq j_{1}, j_{2} \leq n} \frac{(-1)^{j_{1}+j_{2}}\left(s_{j_{1}}(s)\right)^{n-1}\left(s_{j_{1}+j_{2}-1}(s)\right)^{n-1} w_{2}\left(s_{j_{1}}(s)\right) w_{1}\left(s_{j_{1}+j_{2}-1}(s)\right)}{\left(j_{1}-1\right) !\left(n-j_{1}\right) !\left(j_{2}-1\right) !\left(n-j_{2}\right) ! \tau^{2 n-2}} u\left(s_{j_{1}+j_{2}-1}(s)\right),
\end{aligned}
$$

where $w_{1}, w_{2}$ are fixed functions on $[r,+\infty)$, and $u$ is a test function on $[r,+\infty)$. In addition,

$$
s_{j_{1}+j_{2}-1}(s+\tau)=s_{j_{1}+j_{2}}(s), 1 \leq j_{1}, j_{2} \leq n .
$$

Formulas (67), (68) explain that formula (64) involves a(s) exactly in $2 n$ points $s_{j}$ of (60)(62), $j=1, \ldots, 2 n$. Actually, formula (67) explains that computing $a_{2}(s)$ via (65) requires a $(s)$ in $2 n-1$ points $s_{j}$ of (60)-(62), $j=1, \ldots, 2 n-1$; formulas (68), (67), (64) explain that computing $a_{2}(s+\tau)$ requires $a(s)$ in shifted $2 n-1$ points $s_{j}$ of (60)-(62), $j=2, \ldots, 2 n$.

Theorem 3.2 is proved in Section 5 .

Note that formulas (64)-(66) for finding $f_{1}$ from $a(s)$ coincide with formulas (53)-(55) for the case when $N=n=1, \sigma=\tau$.

Note also that Theorem 3.2 is used in the proofs of Propositions 3.1 and 3.2; see Subsections 3.3, 3.4 and Section 6. 


\subsection{Formulas for finding $f_{1}$ from $a$ at $3 n-1$ points for $d=3$}

In this Subsection we give formulas for finding $f_{1}$ up to $\mathcal{O}\left(s^{-n}\right)$, as $s \rightarrow+\infty$, from $a\left(s_{j}(s)\right), j=$ $1, \ldots, 3 n-1$, where $a(s)$ is defined by $(45), s_{j}=s_{j}(s)$ are the points of $(69)-(71)$. We use that $a(s)$ satisfies (46). In constrast to Theorem 3.1, we do not assume that differences between some of these points are multiple to $\pi / \kappa$. In addition, in constrast to Theorem 3.2 , we do not assume that $a$ is reduced to the linearised $a$ of the form (59). On the other hand, the disadvantage of approximate finding $f_{1}$ mentioned above, is that this finding uses $a$ at $3 n-1$ points, in contrast to $2 n$ points of Theorems 3.1 and 3.2 .

We consider $3 n-1$ points $s_{j} \in[r,+\infty), j=1, \ldots, 3 n-1$, of the form

$$
\begin{aligned}
& s_{j}=s_{j}(s)=s+\tau_{j}, j=1, \ldots, 3 n-1, \\
& \tau_{j}=(j-1) \tau, j=1, \ldots, 3 n-1, \\
& s>r, \tau>0, \tau \neq 0\left(\bmod \frac{\pi}{\kappa}\right) .
\end{aligned}
$$

Proposition 3.1. Let $a=a(s)$ satisfy (46) for some $N \geq 2 n-1, n \in \mathbb{N}$. Let $\Sigma_{n, \tau}$ be defined by (63). Then $a(s)$ at $3 n-1$ points $s_{j}$ of (69)-(71) approximately determines $f_{1}$ as follows:

$$
\begin{aligned}
& f_{1}=C_{1}(\kappa, \tau, n)\left(\tau^{n-1}\left(e^{-2 i \tau \kappa} a_{2}(s)-a_{2}(s+\tau)\right)+\mathcal{O}\left(s^{-n}\right)\right), s \rightarrow+\infty, \\
& a_{2}(s):=\Sigma_{n, \tau} a_{1}(s), a_{1}(s):=\frac{e^{-2 i s \kappa}}{s^{n-1}} \Sigma_{n, \tau}\left(e^{i s \kappa} a_{0}\right)(s), \\
& a_{0}(s):=\frac{1}{s^{n-1}} \Sigma_{n, \tau} a(s), \\
& C_{1}(\kappa, \tau, n)=\frac{(n-1) ! \tau^{n-1}}{\left(e^{i \tau \kappa}-1\right)^{n-1}} C(\kappa, \tau, n),
\end{aligned}
$$

where $C(\kappa, \tau, n)$ is given by (66).

Remark 3.3. For $\Sigma_{n, \tau}$ defined by (63) and $3 n-1$ points $s_{j}$ of (69)-(71), we have that

$$
\begin{aligned}
& \Sigma_{n, \tau}\left(w_{3} \Sigma_{n, \tau}\left(w_{2} \Sigma_{n, \tau} w_{1} u\right)\right)(s)= \\
& =\sum_{1 \leq j_{1}, j_{2}, j_{3} \leq n} \frac{(-1)^{n+j_{1}+j_{2}+j_{3}}\left(s_{j_{1}}(s)\right)^{n-1}\left(s_{j_{1}+j_{2}-1}(s)\right)^{n-1}\left(s_{j_{1}+j_{2}+j_{3}-2}(s)\right)^{n-1}}{\left(j_{1}-1\right) !\left(n-j_{1}\right) !\left(j_{2}-1\right) !\left(n-j_{2}\right) !\left(j_{3}-1\right) !\left(n-j_{3}\right) ! \tau^{3 n-3}} \times \\
& w_{3}\left(s_{j_{1}}(s)\right) w_{2}\left(s_{j_{1}+j_{2}-1}(s)\right) w_{1}\left(s_{j_{1}+j_{2}+j_{3}-2}(s)\right) u\left(s_{j_{1}+j_{2}+j_{3}-2}(s)\right),
\end{aligned}
$$

where $w_{1}, w_{2}, w_{3}$ are fixed functions on $[r,+\infty)$, and $u$ is a test function on $[r,+\infty)$. In addition,

$$
s_{j_{1}+j_{2}+j_{3}-2}(s+\tau)=s_{j_{1}+j_{2}+j_{3}-1}(s), 1 \leq j_{1}, j_{2}, j_{3} \leq n .
$$

Formulas (76), (77) explain that formula (72) involves a(s) exactly in $3 n-1$ points $s_{j}$ of (69) $-(71), j=1, \ldots, 3 n-1$. Actually, formulas (73), (74) and (76) explain that computing $a_{2}(s)$ via (73), (74) involves a(s) in $3 n-2$ points $s_{j}$ of (69)-(71), $j=1, \ldots, 3 n-2$; formulas (76), (77) explain that computing $a_{2}(s+\tau)$ via formulas (73), (74) requires a $(s)$ in shifted $3 n-2$ points $s_{j}$ of (69)-(r1), $j=2, \ldots, 3 n-1$.

Proposition 3.1 is proved in Section 6.

Note that formulas (45), (69)-(75) for finding $f_{1}$ from $a$ remain valid for any odd $d \geq 3$, in a similar way with formulas (45), (48)-(57).

Note also that formulas $(72)-(75)$ for finding $f_{1}$ from $a(s)$ coincide with formulas $(53)-(55)$ and with formulas (64)-(66) for the case when $N=n=1, \sigma=\tau$. For this case these formulas were given in [26], [28]. 


\subsection{Formulas for finding $f_{1}$ from $a$ at $3 n$ points for $d=2$}

For $d=2$, the function $a(x, k)$ of formulas $(7),(28)-(32)$ at fixed $k$ and $\hat{x}=x /|x|$ can be written as $a(s)$ defined according to (45), where

$$
\begin{aligned}
& a(s)=\sum_{j=1}^{N} \frac{e^{i s \kappa} f_{j}+e^{-i s \kappa} \bar{f}_{j}}{s^{j-1}}+\sum_{j=1}^{N} \frac{h_{j}}{s^{j-1 / 2}}+\mathcal{O}\left(\frac{1}{s^{N}}\right), s \rightarrow+\infty, \\
& h_{j}=\sum_{k=1}^{j} f_{k} \bar{f}_{j-k+1}, j=1, \ldots, N,
\end{aligned}
$$

where $s=|x|, \kappa=|k|-k \hat{x}$, and $\kappa>0$ if $\hat{x}=x /|x| \neq \hat{k}=k /|k|$.

Proceeding from this motivation, we consider arbitrary functions $a=a(s), s \in[r,+\infty), r>0$, such that formula (78) holds for some fixed $\kappa>0$ and some complex numbers $f_{j}, j=1, \ldots, N$, and $h_{j}, j=1, \ldots, N$.

In this Subsection we give formulas for finding $f_{1}$ up to $\mathcal{O}\left(s^{-n}\right)$, as $s \rightarrow+\infty$, from $a\left(s_{j}(s)\right), j=$ $1, \ldots, 3 n$, where $a(s)$ is of the form $(78), s_{j}=s_{j}(s)$ are the points of (80)-(82). In constrast to Theorem 3.2, we do not assume that $a$ is reduced to the linearised $a$ of the form (59). On the other hand, the disadvantage of approximate finding $f_{1}$ mentioned above, is that this finding uses $a$ at $3 n$ points, in contrast to $2 n$ points of Theorem 3.2 .

We consider $3 n$ points $s_{j} \in[r,+\infty), j=1, \ldots, 3 n$ of the form

$$
\begin{aligned}
& s_{j}(s)=s+\tau_{j}, j=1, \ldots, 3 n, \\
& \tau_{j}=(j-1) \tau, j=1, \ldots, 3 n, \\
& s \geq r>0, \tau>0, \tau \neq 0\left(\bmod \frac{\pi}{\kappa}\right) .
\end{aligned}
$$

Proposition 3.2. Let a(s) satisfy (78) for some $N \geq 2 n+1, n \in \mathbb{N}$. Let $\Sigma_{n, \tau}$ be defined by (63). Then $a(s)$ at $3 n$ points $s_{j}$ of (80)-(82) approximately determines $f_{1}$ as follows:

$$
\begin{aligned}
& f_{1}=C_{2}(\kappa, \tau, n)\left(\tau^{n-1}\left(e^{-2 i \tau \kappa} a_{3}(s)-a_{3}(s+\tau)\right)+\mathcal{O}\left(s^{-n}\right)\right), s \rightarrow+\infty \\
& a_{3}(s):=\Sigma_{n, \tau} a_{2}(s), a_{2}(s):=\frac{e^{-2 i s \kappa}}{s^{n-1}} \Sigma_{n, \tau}\left(e^{i s \kappa} a_{1}\right)(s), a_{1}(s)=\frac{1}{s^{n-1 / 2}} \Sigma_{n+1, \tau} a_{0}(s), a_{0}(s)=a(s) / \sqrt{s} \\
& C_{2}(\kappa, \tau, n)=\frac{n !(n-1) ! \tau^{n}}{\left(e^{i \tau \kappa}-1\right)^{n}\left(e^{2 i \tau \kappa}-1\right)^{n-1}\left(e^{-2 i \tau \kappa}-1\right)} .
\end{aligned}
$$

Remark 3.4. For $\Sigma_{n, \tau}$ defined by (63) and $3 n$ points $s_{j}$ of (80)-(82), we have that

$$
\begin{aligned}
& \Sigma_{n, \tau}\left(w_{3} \Sigma_{n, \tau}\left(w_{2} \Sigma_{n+1, \tau} w_{1} u\right)\right)(s)= \\
& =\sum_{1 \leq j_{1}, j_{2} \leq n} \sum_{1 \leq j_{3} \leq n+1} \frac{(-1)^{n+j_{1}+j_{2}+j_{3}}\left(s_{j_{1}}(s)\right)^{n-1}\left(s_{j_{1}+j_{2}-1}(s)\right)^{n-1}\left(s_{j_{1}+j_{2}+j_{3}-2}(s)\right)^{n-1}}{\left(j_{1}-1\right) !\left(n-j_{1}\right) !\left(j_{2}-1\right) !\left(n-j_{2}\right) !\left(j_{3}-1\right) !\left(n-j_{3}\right) ! \tau^{3 n-3}} \times \\
& \times w_{3}\left(s_{j_{1}}(s)\right) w_{2}\left(s_{j_{1}+j_{2}-1}(s)\right) w_{1}\left(s_{j_{1}+j_{2}+j_{3}-2}(s)\right) u\left(s_{j_{1}+j_{2}+j_{3}-2}(s)\right),
\end{aligned}
$$

where $w_{1}, w_{2}, w_{3}$ are fixed functions on $[r,+\infty)$, and $u$ is a test function on $[r,+\infty)$. In addition,

$$
s_{j_{1}+j_{2}+j_{3}-2}(s+\tau)=s_{j_{1}+j_{2}+j_{3}-1}(s), 1 \leq j_{1}, j_{2}, j_{3} \leq n .
$$

Formulas (86), (87) explain that formulas (83) involves a(s) exactly in $3 n$ points $s_{j}$ of (80)-(82), $j=1, \ldots, 3 n$. Actually, formulas (86) and (84) explain that computing $a_{2}(s)$ via (84) involves a $(s)$ in $3 n-1$ points $s_{j}$ of (80)-(82), $j=1, \ldots, 3 n-1$; formulas (86), (87) explain that computing $a_{2}(s+\tau)$ via formulas (84) involves $a(s)$ in $3 n-1$ shifted points $s_{j}$ of (80)-(82), $j=2, \ldots, 3 n$.

Proposition 3.2 is proved in Section 6 .

Note that formulas (45), (80)-(85) for finding $f_{1}$ from $a$ remain valid for any even $d \geq 2$, in a similar way with formulas (45), (48)-(57), (69)-(75) for odd $d \geq 3$. 


\section{Proof of Theorem 3.1}

Let

$$
\vec{\tau}_{1}=\left(\tau_{1,1}, \ldots, \tau_{1, n}\right), \vec{\tau}_{2}=\left(\tau_{2,1}, \ldots, \tau_{2, n}\right)
$$

Let $y_{j}\left(s, \vec{\tau}_{1}\right), y_{j}\left(s, \vec{\tau}_{2}\right), j=1, \ldots, n$, be defined according to $(41),(42)$. We have that

$$
a_{1}(s)=\sum_{j=1}^{n} y_{j}\left(s, \vec{\tau}_{1}\right) a\left(s_{1, j}(s)\right), a_{2}(s)=\sum_{j=1}^{n} y_{j}\left(s, \vec{\tau}_{2}\right) a\left(s_{2, j}(s)\right) .
$$

Note that

$$
\begin{aligned}
& e^{i s_{1, j}(s) \kappa}=e^{i s \kappa} e^{i \nu_{1, j} \tau \kappa}=e^{i s \kappa}, \quad j=1, \ldots, n, \\
& e^{i s_{2, j}(s) \kappa}=e^{i(s+\sigma) \kappa} e^{i \nu_{2, j} \tau \kappa}=e^{i(s+\sigma) \kappa}, \quad j=1, \ldots, n .
\end{aligned}
$$

Using formulas (41)-(44), (46), (89), (90) and the assumption that $N \geq 2 n-1$, we obtain that:

$$
\begin{aligned}
& a_{1}(s)=\sum_{j=1}^{n} y_{j}\left(s, \vec{\tau}_{1}\right)\left(\sum_{m=1}^{N} \frac{e^{i s_{1, j}(s) \kappa} f_{m}+e^{-i s_{1, j}(s) \kappa} \bar{f}_{m}+h_{m}}{\left(s+\tau_{1, j}\right)^{m-1}}+\mathcal{O}\left(s^{-N}\right)\right)= \\
& =\sum_{m=1}^{N}\left(e^{i s \kappa} f_{m}+e^{-i s \kappa} \bar{f}_{m}+h_{m}\right) \sum_{j=1}^{n} \frac{y_{j}\left(s, \vec{\tau}_{1}\right)}{\left(s+\tau_{1, j}\right)^{m-1}}+\mathcal{O}\left(s^{-n}\right)= \\
& =e^{i s \kappa} f_{1}+e^{-i s \kappa} \bar{f}_{1}+\mathcal{O}\left(s^{-n}\right), \text { as } s \rightarrow+\infty ; \\
& a_{2}(s)=\sum_{j=1}^{n} y_{j}\left(s, \vec{\tau}_{2}\right)\left(\sum_{m=1}^{N} \frac{e^{i s_{2, j}(s) \kappa} f_{m}+e^{-i s_{2, j}(s) \kappa} \bar{f}_{m}+h_{m}}{\left(s+\sigma+\tau_{2, j}\right)^{m-1}}+\mathcal{O}\left(s^{-N}\right)\right)= \\
& =\sum_{m=1}^{N}\left(e^{i(s+\sigma) \kappa} f_{m}+e^{-i(s+\sigma) \kappa} \bar{f}_{m}+h_{m}\right) \sum_{j=1}^{n} \frac{y_{j}\left(s, \vec{\tau}_{2}\right)}{\left(s+\sigma+\tau_{2, j}\right)^{m-1}}+\mathcal{O}\left(s^{-n}\right)= \\
& =e^{i(s+\sigma) \kappa} f_{1}+e^{-i(s+\sigma) \kappa} \bar{f}_{1}+\mathcal{O}\left(s^{-n}\right), \text { as } s \rightarrow+\infty .
\end{aligned}
$$

We consider formulas (91), (92) as a linear system for approximate finding $f_{1}, \bar{f}_{1}$ from $a_{1}(s), a_{2}(s)$, where $s$ is sufficiently large.

If $\sigma>0, \sigma \neq 0(\bmod \pi / \kappa)$, then from $(91),(92)$ we obtain that

$$
\left(\begin{array}{c}
f_{1} \\
f_{1}
\end{array}\right)=\frac{1}{e^{-i \sigma \kappa}-e^{i \sigma \kappa}}\left(\begin{array}{cc}
e^{-i(s+\sigma) \kappa} & -e^{-i s \kappa} \\
-e^{i(s+\sigma) \kappa} & e^{i s \kappa}
\end{array}\right)\left(\begin{array}{ll}
a_{1}(s)+ & \mathcal{O}\left(s^{-n}\right) \\
a_{2}(s)+ & \mathcal{O}\left(s^{-n}\right)
\end{array}\right) .
$$

Formula (53) follows from (93).

Theorem 3.1 is proved.

\section{Proof of Theorem 3.2}

Lemma 5.1. Let $\Sigma_{n, \tau}$ be defined via (63). Let

$$
u(s)=\sum_{j=1}^{N} \frac{e^{i s \kappa} f_{j}}{s^{j-1}}, s \in[r,+\infty),
$$


for some fixed $\kappa \in \mathbb{R}$ and some complex numbers $f_{j}, j=1, \ldots, N$, where $N \geq 2 n-1$. Then:

$$
\begin{aligned}
& \Sigma_{n, \tau} u(s)=s^{n-1} \sum_{m=1}^{2 n-1} \frac{e^{i s \kappa} f_{m, \kappa}}{s^{m-1}}+\mathcal{O}\left(s^{-n}\right), s \in[r,+\infty), \\
& f_{m, \kappa}=f_{m, \kappa, n, \tau}\left(f_{1}, \ldots, f_{m}\right)=\sum_{j_{2}=1}^{m} \frac{f_{j_{2}} C_{n-j_{2}}^{m-j_{2}}}{\tau^{n-1}} \sum_{j_{1}=1}^{n} \frac{(-1)^{n-j_{1}} \tau_{j_{1}}^{m-j_{2}} e^{i \tau_{j_{1}} \kappa}}{\left(j_{1}-1\right) !\left(n-j_{1}\right) !}, \forall m=1, \ldots, 2 n-1,
\end{aligned}
$$

where $C_{\beta}^{\alpha}$ denote the binomial coefficients,

$$
\begin{aligned}
f_{1, \kappa} & =\frac{\left(e^{i \tau \kappa}-1\right)^{n-1}}{(n-1) ! \tau^{n-1}} f_{1}, \\
f_{n, \kappa} & =\frac{\left(e^{i \tau \kappa}-1\right)^{n-1}}{(n-1) ! \tau^{n-1}} f_{n} \text { if } f_{1}=\ldots=f_{n-1}=0 .
\end{aligned}
$$

Proof of Lemma 5.1. Using (63), (94), we obtain that

$$
\begin{aligned}
& \Sigma_{n, \tau} u(s)=\sum_{j_{1}=1}^{n} \sum_{j_{2}=1}^{N} \frac{(-1)^{n-j_{1}}\left(s+\tau_{j_{1}}\right)^{n-1}}{\left(j_{1}-1\right) !\left(n-j_{1}\right) ! \tau^{n-1}} \frac{e^{i\left(s+\tau_{j_{1}}\right) \kappa} f_{j_{2}}}{\left(s+\tau_{j_{1}}\right)^{j_{2}-1}}= \\
& =\frac{e^{i s \kappa}}{\tau^{n-1}} \sum_{j_{2}=1}^{N} f_{j_{2}} \sum_{j_{1}=1}^{n} \frac{(-1)^{n-j_{1}}\left(s+\tau_{j_{1}}\right)^{n-j_{2}} e^{i \tau_{j_{1}} \kappa}}{\left(j_{1}-1\right) !\left(n-j_{1}\right) !}, s \in[r,+\infty) .
\end{aligned}
$$

Note that

$$
\begin{aligned}
& \left(s+\tau_{j_{1}}\right)^{n-j_{2}}=\sum_{j_{3}=0}^{n-j_{2}} C_{n-j_{2}}^{j_{3}} s^{n-j_{2}-j_{3}} \tau_{j_{1}}^{j_{3}}=\sum_{j_{3}=0}^{2 n-j_{2}-1} C_{n-j_{2}}^{j_{3}} \tau_{j_{1}}^{j_{3}} s^{n-j_{2}-j_{3}}, n-j_{2} \geq 0, \\
& \left(s+\tau_{j_{1}}\right)^{n-j_{2}}=\frac{1}{s^{j_{2}-n}} \sum_{j_{3}=0}^{\infty} C_{n-j_{2}}^{j_{3}} \tau_{j_{1}}^{j_{3}} s^{-j_{3}}=\sum_{j_{3}=0}^{2 n-j_{2}-1} C_{n-j_{2}}^{j_{3}} \tau_{j_{1}}^{j_{3}} s^{n-j_{2}-j_{3}}+\mathcal{O}\left(s^{-n}\right), n-j_{2}<0,
\end{aligned}
$$

where $C_{\beta}^{\alpha}$ are the binomial coefficients; we used in (101) that $C_{\beta}^{\alpha}=0$, for $\alpha>\beta$, if $\beta \in \mathbb{N}$. Using (99), (101), (102) we obtain that

$$
\begin{aligned}
& \frac{\Sigma_{n, \tau} u(s)}{s^{n-1}}=\frac{e^{i s \kappa}}{\tau^{n-1}} \sum_{j_{2}=1}^{N} f_{j_{2}} \sum_{j_{1}=1}^{n} \frac{(-1)^{n-j_{1}} \sum_{j_{3}=0}^{2 n-j_{2}-1}\left(C_{n-j_{2}}^{j_{3}} \tau_{j_{1}}^{j_{3}} / s^{j_{2}+j_{3}-1}\right) e^{i \tau_{j_{1}} \kappa}}{\left(j_{1}-1\right) !\left(n-j_{1}\right) !}+\mathcal{O}\left(s^{-2 n+1}\right)= \\
& =\frac{e^{i s \kappa}}{\tau^{n-1}} \sum_{j_{2}=1}^{2 n-1} f_{j_{2}} \sum_{j_{1}=1}^{n} \frac{(-1)^{n-j_{1}} \sum_{j_{3}=0}^{2 n-j_{2}-1}\left(C_{n-j_{2}}^{j_{3}} \tau_{j_{1}}^{j_{3}} / s^{j_{2}+j_{3}-1}\right) e^{i \tau_{j_{1}} \kappa}}{\left(j_{1}-1\right) !\left(n-j_{1}\right) !}+\mathcal{O}\left(s^{-2 n+1}\right)= \\
& =\frac{e^{i s \kappa}}{\tau^{n-1}} \sum_{j_{3}=0}^{2 n-2} \sum_{j_{2}=1}^{2 n-j_{3}-1} \frac{f_{j_{2}} C_{n-j_{2}}^{j_{3}}}{s^{j_{2}+j_{3}-1}} \sum_{j_{1}=1}^{n} \frac{(-1)^{n-j_{1}} \tau_{j_{1}}^{j_{3}} e^{i \tau_{j_{1}} \kappa}}{\left(j_{1}-1\right) !\left(n-j_{1}\right) !}+\mathcal{O}\left(s^{-2 n+1}\right) \stackrel{m=j_{2}+j_{3}}{=} \\
& =\sum_{m=1}^{2 n-1} \frac{e^{i s \kappa}}{s^{m-1}} \sum_{j_{2}=1}^{m} \frac{f_{j_{2}} C_{n-j_{2}}^{m-j_{2}}}{\tau^{n-1}} \sum_{j_{1}=1}^{n} \frac{(-1)^{n-j_{1}} \tau_{j_{1}}^{m-j_{2}} e^{i \tau_{j_{1}} \kappa}}{\left(j_{1}-1\right) !\left(n-j_{1}\right) !}+\mathcal{O}\left(s^{-2 n+1}\right)=\sum_{m=1}^{2 n-1} \frac{e^{i s \kappa} f_{m, \kappa}}{s^{m-1}}+\mathcal{O}\left(s^{-2 n+1}\right),
\end{aligned}
$$

where

$$
\begin{aligned}
f_{m, \kappa} & =\sum_{j_{2}=1}^{m} \frac{f_{j_{2}} C_{n-j_{2}}^{m-j_{2}}}{\tau^{n-1}} \sum_{j_{1}=1}^{n} \frac{(-1)^{n-j_{1}} \tau_{j_{1}}^{m-j_{2}} e^{i \tau_{j_{1}} \kappa}}{\left(j_{1}-1\right) !\left(n-j_{1}\right) !} \\
f_{1, \kappa} & =\frac{f_{1} C_{n-1}^{0}}{\tau^{n-1}} \sum_{j=1}^{n} \frac{(-1)^{n-j} e^{i \tau(j-1) \kappa}}{(j-1) !(n-j) !}=\frac{\left(e^{i \tau \kappa}-1\right)^{n-1}}{(n-1) ! \tau^{n-1}} f_{1} .
\end{aligned}
$$


Note that in (103) we used the change of variables $\left(j_{1}, j_{2}, j_{3}\right) \rightarrow\left(j_{1}, j_{2}, m\right), m=j_{2}+j_{3}$.

Formulas (95), (97) follows from (103), (104), (105).

In addition, if $f_{1}=\ldots=f_{n-1}=0$, then

$$
f_{n, \kappa}=f_{n} \frac{C_{0}^{0}}{\tau^{n-1}} \sum_{j=1}^{n} \frac{(-1)^{n-j} e^{i(j-1) \tau \kappa}}{(j-1) !(n-j) !}=\frac{\left(e^{i \tau \kappa}-1\right)^{n-1}}{(n-1) ! \tau^{n-1}} f_{n} .
$$

Lemma 5.1 is proved.

The rest of the proof of Theorem 3.2 is as follows. Due to (59), we have that

$$
\begin{aligned}
& e^{i s \kappa} a(s)=u(s)+z(s), \\
& u(s)=\sum_{j=1}^{N} \frac{e^{2 i s \kappa} f_{j}}{s^{j-1}}, \quad z(s)=\sum_{j=1}^{N} \frac{\bar{f}_{j}}{s^{j-1}}+\mathcal{O}\left(s^{-N}\right), \text { as } s \rightarrow+\infty .
\end{aligned}
$$

Due to formulas (40), (42), (44), (63), and the formula for $z$ in (107), we have that

$$
\Sigma_{n, \tau} z(s)=\bar{f}_{1}+\mathcal{O}\left(s^{-n}\right), \text { as } s \rightarrow+\infty .
$$

Due to Lemma 5.1 (with $2 \kappa$ in place of $\kappa$ ) and the formula for $u$ in (107), we have that

$$
\begin{aligned}
& \Sigma_{n, \tau} u(s)=s^{n-1} \sum_{m=1}^{2 n-1} \frac{e^{2 i s \kappa} f_{m, 2 \kappa}}{s^{m-1}}+\mathcal{O}\left(s^{-n}\right), \text { where } \\
& f_{1,2 \kappa}=\frac{\left(e^{2 i \tau \kappa}-1\right)^{n-1}}{(n-1) ! \tau^{n-1}} f_{1} .
\end{aligned}
$$

Due to (107), (108), (109), we have that

$$
\Sigma_{n, \tau}\left(e^{i s \kappa} a\right)(s)=s^{n-1} \sum_{m=1}^{2 n-1} \frac{e^{2 i s \kappa} f_{m, 2 \kappa}}{s^{m-1}}+\mathcal{O}\left(s^{-n}\right)+\bar{f}_{1}+\mathcal{O}\left(s^{-n}\right), \text { as } s \rightarrow+\infty .
$$

The definition of $a_{1}$ in (65) and formula (110) imply that

$$
\begin{aligned}
& a_{1}(s)=u_{1}(s)+z_{1}(s), \text { where } \\
& u_{1}(s)=\frac{e^{-2 i s \kappa} \bar{f}_{1}}{s^{n-1}}, z_{1}(s)=\sum_{m=1}^{2 n-1} \frac{f_{m, 2 \kappa}}{s^{m-1}}+\mathcal{O}\left(s^{-2 n+1}\right), \text { as } s \rightarrow+\infty .
\end{aligned}
$$

Due to formulas (40), (42), (44), (63), and the formula for $z_{1}$ in (111), we have that

$$
\Sigma_{n, \tau} z_{1}(s)=f_{1,2 \kappa}+\mathcal{O}\left(s^{-n}\right), \text { as } s \rightarrow+\infty .
$$

Besides, the following formula holds,

$$
\Sigma_{n, \tau} u_{1}(s)=e^{-2 i s \kappa} \bar{f}_{1,-2 \kappa}, \quad \bar{f}_{1,-2 \kappa}=\frac{\left(e^{-2 i \tau \kappa}-1\right)^{n-1}}{(n-1) ! \tau^{n-1}} \bar{f}_{1} .
$$

Formula (113) follows from formulas (95), (96), (98) of Lemma 5.1, with $(-2 \kappa)$ in place of $\kappa$, $f_{n}=\bar{f}_{1}$, where $\bar{f}_{1}$ is the number in definition of $u_{1}$ in (111).

The definition of $a_{2}$ in (65) and formulas (109), (111)-(113) imply that

$$
\begin{aligned}
& a_{2}(s)=f_{1,2 \kappa}+e^{-2 i s \kappa} \bar{f}_{1,-2 \kappa}+\mathcal{O}\left(s^{-n}\right)= \\
& =\frac{\left(e^{2 i \tau \kappa}-1\right)^{n-1} f_{1}+e^{-2 i s \kappa}\left(e^{-2 i \tau \kappa}-1\right)^{n-1} \bar{f}_{1}}{(n-1) ! \tau^{n-1}}+\mathcal{O}\left(s^{-n}\right), s \rightarrow+\infty .
\end{aligned}
$$


From (114) we obtain that

$$
\begin{aligned}
& \left(e^{2 i \tau \kappa}-1\right)^{n-1} f_{1}+e^{-2 i s \kappa}\left(e^{-2 i \tau \kappa}-1\right)^{n-1} \bar{f}_{1}=(n-1) ! \tau^{n-1} a_{2}(s)+\mathcal{O}\left(s^{-n}\right), s \rightarrow+\infty, \\
& \left(e^{2 i \tau \kappa}-1\right)^{n-1} f_{1}+e^{-2 i(s+\tau) \kappa}\left(e^{-2 i \tau \kappa}-1\right)^{n-1} \bar{f}_{1}=(n-1) ! \tau^{n-1} a_{2}(s+\tau)+\mathcal{O}\left(s^{-n}\right), s \rightarrow+\infty .
\end{aligned}
$$

We consider formulas (115) as a linear system for approximate finding $f_{1}, \bar{f}_{1}$ from $(n-1) ! \tau^{n-1} a_{2}(s),(n-1) ! \tau^{n-1} a_{2}(s+\tau)$, where $s$ is sufficiently large. From this system, for $\tau>0$, $\tau \neq 0(\bmod \pi / \kappa)$, we obtain that

$$
\begin{aligned}
& \left(\begin{array}{c}
f_{1} \\
f_{1}
\end{array}\right)=\frac{1}{\left(e^{2 i \tau \kappa}-1\right)^{n-1}\left(e^{-2 i \tau \kappa}-1\right)^{n-1} e^{-2 i s \kappa}\left(e^{-2 i \tau \kappa}-1\right)} \times \\
& \left(\begin{array}{cc}
e^{-2 i(s+\tau) \kappa}\left(e^{-2 i \tau \kappa}-1\right)^{n-1} & -e^{-2 i s \kappa}\left(e^{-2 i \tau \kappa}-1\right)^{n-1} \\
-\left(e^{2 i \tau \kappa}-1\right)^{n-1} & \left(e^{2 i \tau \kappa}-1\right)^{n-1}
\end{array}\right)\left(\begin{array}{c}
(n-1) ! \tau^{n-1} a_{2}(s)+\mathcal{O}\left(s^{-n}\right) \\
(n-1) ! \tau^{n-1} a_{2}(s+\tau)+\mathcal{O}\left(s^{-n}\right)
\end{array}\right), s \rightarrow+\infty .
\end{aligned}
$$

Formula (116) implies that

$$
f_{1}=\frac{(n-1) ! \tau^{n-1}\left(e^{-2 i \tau \kappa} a_{2}(s)-a_{2}(s+\tau)\right)+\mathcal{O}\left(s^{-n}\right)}{\left(e^{2 i \tau \kappa}-1\right)^{n-1}\left(e^{-2 i \tau \kappa}-1\right)}, s \rightarrow+\infty .
$$

This completes the proof of Theorem 3.2.

\section{Proofs of Propositions 3.1 and 3.2}

Proof of Proposition 3.1. The following formula holds:

$$
\Sigma_{n, \tau} a(s)=s^{n-1} \sum_{m=1}^{n} \frac{e^{i s \kappa} f_{m, \kappa}+e^{-i s \kappa} \bar{f}_{m,-\kappa}}{s^{m-1}}+\mathcal{O}\left(s^{-n}\right), s \rightarrow+\infty,
$$

where $f_{m, \kappa}$ and $\bar{f}_{m,-\kappa}$ are defined according to (96) in terms of $f_{1}, \ldots, f_{m}$ and $\bar{f}_{1}, \ldots, \bar{f}_{m}$, respectively. In particular, according to (97), we have that

$$
f_{1, \kappa}=\frac{\left(e^{i \tau \kappa}-1\right)^{n-1}}{(n-1) ! \tau^{n-1}} f_{1}, \bar{f}_{1,-\kappa}=\frac{\left(e^{-i \tau \kappa}-1\right)^{n-1}}{(n-1) ! \tau^{n-1}} \bar{f}_{1} .
$$

Formula (118) follows from formula (46), Lemma 5.1 and formulas (40), (42), (44), (63).

Formula (72), where $a_{2}$ is defined by (73), (74), follows from formulas (118), (119), and Theorem 3.2 .

Proposition 3.1 is proved.

Proof of Proposition 3.2. Assume that

$$
n \tau / s<1, \text { where } s \geq r>0 .
$$

From the definition of $a_{0}$ in (84) and formula (78) we have that

$$
a_{0}(s)=\sum_{j=1}^{N} \frac{e^{i s \kappa} f_{j}}{s^{j-1 / 2}}+\sum_{j=1}^{N} \frac{e^{-i s \kappa} \bar{f}_{j}}{s^{j-1 / 2}}+\left(\sum_{j=2}^{N} \frac{h_{j-1}}{s^{j-1}}+\mathcal{O}\left(\frac{1}{s^{N}}\right)\right)=: a_{0,1}(s)+a_{0,2}(s)+a_{0,3}(s) .
$$

Using that $N \geq 2 n+1$ and formulas (38), (40) with $n+1$ in place of $n$, we obtain that

$$
\Sigma_{n+1, \tau} a_{0,3}(s)=\mathcal{O}\left(s^{-(n+1)}\right), s \rightarrow+\infty .
$$


We also have that

$$
\begin{aligned}
& \Sigma_{n+1, \tau} a_{0,1}(s)=\sum_{j=1}^{n+1} \frac{(-1)^{n+1-j}\left(s+\tau_{j}\right)^{n} \sum_{k=1}^{N} e^{i\left(s+\tau_{j}\right) \kappa} f_{k} /\left(s+\tau_{j}\right)^{k-1 / 2}}{(j-1) !(n+1-j) ! \tau^{n}}= \\
& =e^{i s \kappa} \sum_{j=1}^{n+1} \sum_{k=1}^{N} \frac{(-1)^{n+1-j}\left(s+\tau_{j}\right)^{n-k+1 / 2} e^{i(j-1) \tau \kappa} f_{k}}{(j-1) !(n+1-j) ! \tau^{n}} .
\end{aligned}
$$

Note that

$$
\begin{aligned}
& \frac{\left(s+\tau_{j}\right)^{n-k+1 / 2}}{s^{n-1 / 2}}=\frac{\left(1+\tau_{j} / s\right)^{n-k+1 / 2}}{s^{k-1}} \\
& \left(1+\tau_{j} / s\right)^{n-k+1 / 2}=\sum_{l=0}^{2 n} C_{n-k+1 / 2}^{l}\left(\frac{\tau_{j}}{s}\right)^{l}+\mathcal{O}\left(s^{-2 n-1}\right), s \rightarrow+\infty,
\end{aligned}
$$

where in (125) we used assumption (120) and that $\tau_{j}=(j-1) \tau, j=1, \ldots, n+1$. Using that $N \geq 2 n+1$ and formulas (123), (124), (125), we obtain that

$$
\begin{aligned}
& \Sigma_{n+1, \tau} a_{0,1}(s) / s^{n-1 / 2}=e^{i s \kappa} \sum_{j=1}^{n+1} \sum_{k=1}^{N} \frac{(-1)^{n+1-j}(1+(j-1) \tau / s)^{n-k+1 / 2} e^{i(j-1) \tau \kappa} f_{k}}{(j-1) !(n+1-j) ! \tau^{n} s^{k-1}}= \\
& =\frac{e^{i s \kappa}}{\tau^{n}} \sum_{j=1}^{n+1} \sum_{k=1}^{N}\left(\sum_{l=0}^{2 n} \frac{(-1)^{n+1-j} C_{n-k+1 / 2}^{l}\left(\frac{(j-1) \tau}{s}\right)^{l} e^{i(j-1) \tau \kappa} f_{k}}{(j-1) !(n+1-j) ! s^{k-1}}+\mathcal{O}\left(s^{-2 n-1}\right)\right)= \\
& =\frac{e^{i s \kappa}}{\tau^{n}} \sum_{j=0}^{n} \sum_{k=1}^{2 n+1} \sum_{l=0}^{2 n} \frac{(-1)^{n-j} C_{n-k+1 / 2}^{l}(j \tau)^{l} e^{i j \tau \kappa} f_{k}}{j !(n-j) ! s^{k-1+l}}+\mathcal{O}\left(s^{-2 n-1}\right) \stackrel{l=m-k}{=} \\
& =\frac{e^{i s \kappa}}{\tau^{n}} \sum_{m=1}^{2 n+1} \sum_{k=1}^{m} \sum_{j=0}^{n} \frac{(-1)^{n-j} C_{n-k+1 / 2}^{m-k}(j \tau)^{m-k} e^{i j \tau \kappa}}{j !(n-j) ! s^{m-1}} f_{k}+\mathcal{O}\left(s^{-2 n-1}\right)= \\
& =\frac{1}{\tau^{n}} \sum_{m=1}^{2 n+1} \frac{e^{i s \kappa}}{s^{m-1}} \sum_{k=1}^{m} f_{k} C_{n-k+1 / 2}^{m-k} \sum_{j=0}^{n} \frac{(-1)^{n-j}(j \tau)^{m-k} e^{i j \tau \kappa}}{j !(n-j) !}+\mathcal{O}\left(s^{-2 n-1}\right)= \\
& =\sum_{m=1}^{2 n+1} \frac{e^{i s \kappa} f_{m, \kappa}}{s^{m-1}}+\mathcal{O}\left(s^{-2 n-1}\right), s \rightarrow+\infty,
\end{aligned}
$$

where

$$
\begin{aligned}
& f_{m, \kappa}=\frac{1}{\tau^{n}} \sum_{k=1}^{m} f_{k} C_{n-k+1 / 2}^{m-k} \sum_{j=0}^{n} \frac{(-1)^{n-j}(j \tau)^{m-k} e^{i j \tau \kappa}}{j !(n-j) !}, m=1, \ldots, 2 n+1, \\
& f_{1, \kappa}=\frac{f_{1}}{\tau^{n}} \sum_{j=0}^{n} \frac{(-1)^{n-j} e^{i j \tau \kappa}}{j !(n-j) !}=\frac{\left(e^{i \tau \kappa}-1\right)^{n}}{\tau^{n} n !} f_{1}, \quad \text { where } \frac{\left(e^{i \tau \kappa}-1\right)^{n}}{\tau^{n} n !} \neq 0 .
\end{aligned}
$$

Note that in (126) we used the change of variables $(j, k, l) \rightarrow(j, k, m), l=m-k$.

In (128) we have that $e^{i \tau \kappa} \neq 1$ due to (82). In a similar way with (126) we also have that

$$
\Sigma_{n+1, \tau} a_{0,2}(s) / s^{n-1 / 2}=\sum_{m=1}^{2 n+1} \frac{e^{-i s \kappa} \overline{f_{m, \kappa}}}{s^{m-1}}+\mathcal{O}\left(s^{-2 n-1}\right), s \rightarrow+\infty .
$$


From formulas (122), (126), (129) we obtain that

$$
\begin{aligned}
& a_{1}(s):=\frac{\Sigma_{n+1, \tau} a_{0}(s)}{s^{n-1 / 2}}=\sum_{j=1}^{2 n+1} \frac{e^{i s \kappa} f_{j, \kappa}+e^{-i s \kappa} \overline{f_{j, \kappa}}}{s^{j-1}}+\mathcal{O}\left(s^{-2 n-1}\right)+\mathcal{O}\left(s^{-2 n-1 / 2}\right)= \\
& =\sum_{j=1}^{2 n+1} \frac{e^{i s \kappa} f_{j, \kappa}+e^{-i s \kappa} \overline{f_{j, \kappa}}}{s^{j-1}}+\mathcal{O}\left(s^{-2 n-1 / 2}\right)=\sum_{j=1}^{2 n-1} \frac{e^{i s \kappa} f_{j, \kappa}+e^{-i s \kappa} \overline{f_{j, \kappa}}}{s^{j-1}}+\mathcal{O}\left(s^{-2 n+1}\right), s \rightarrow+\infty .
\end{aligned}
$$

From (130) one can see that the function $a_{1}(s)$ satisfies the assumptions of Theorem 3.2 for $N=2 n-1$. Therefore, applying Theorem 3.2 to the function $a_{1}(s)$ we reconstruct $f_{1, \kappa}$ via formulas

$$
\begin{aligned}
& f_{1, \kappa}=C(\kappa, \tau, n)\left(\tau^{n-1}\left(e^{-2 i \tau \kappa} a_{3}(s)-a_{3}(s+\tau)\right)+\mathcal{O}\left(s^{-n}\right)\right), s \rightarrow+\infty, \\
& a_{3}(s):=\Sigma_{n, \tau} a_{2}(s), a_{2}(s):=\frac{e^{-2 i s \kappa}}{s^{n-1}} \Sigma_{n, \tau}\left(e^{i s \kappa} a_{1}\right)(s), \\
& C(\kappa, \tau, n):=\frac{(n-1) !}{\left(e^{2 i \tau \kappa}-1\right)^{n-1}\left(e^{-2 i \tau \kappa}-1\right)} .
\end{aligned}
$$

In addition, formula (128) implies that

$$
f_{1}=\frac{n ! \tau^{n} f_{1, \kappa}}{\left(e^{i \tau \kappa}-1\right)^{n}}
$$

Formulas (83), (84), (85) follow from formulas (131)-(134) and the definitions of $a_{1}(s), a_{0}(s)$. This completes the proof of Proposition 3.2.

\section{$7 \quad$ Sketch of proof of formulas $(23)-(25)$}

We will use that

$$
\begin{aligned}
& G^{+}(x-y, k)=G_{n}^{+}(x-y, k)+\rho_{n}(y, x,|k|), \\
& G_{n}^{+}(x-y, k)=\frac{e^{i|k||x|}}{|x|^{(d-1) / 2}}\left(\sum_{j=1}^{n} \frac{g_{j}(y, \hat{x},|k|)}{|x|^{j-1}}\right), \\
& g_{1}(y, \hat{x},|k|)=e^{-i|k| y \hat{x}}, \rho_{n}(y, x,|k|):=\frac{e^{i|k||x|}}{|x|^{(d-1) / 2}} \varepsilon_{n}(y, x,|k|), \\
& \varepsilon_{n}(y, x,|k|)=\mathcal{O}\left(\frac{1}{|x|^{n}}\right),|x| \rightarrow+\infty, \text { uniformly in } y \in D,
\end{aligned}
$$

where $G^{+}$is defined in (16), $\hat{x}=x /|x|$. The Lippman-Schwinger integral equation (16) and formula (135) imply formulas (19), (23), (24), where

$$
(2 \pi)^{-d} c(d,|k|) \varphi_{j}(y, l)=g_{j}(y, \hat{l},|k|), \hat{l}=l /|l| .
$$

The recurrent relations (25) can be proved using also that:

$$
\begin{aligned}
& \left(\Delta_{x}+k^{2}\right) G^{+}(x-y, k)=0, \text { for } x \in \mathbb{R}^{d} \backslash D, y \in D \\
& \Delta_{x}=\frac{\partial^{2}}{\partial r^{2}}+\frac{d-1}{r} \frac{\partial}{\partial r}+\frac{1}{r^{2}} \Delta_{S}
\end{aligned}
$$

where $r=|x|, \Delta_{S}$ is the Beltrami-Laplace operator with respect to $\hat{x} \in \mathbb{S}^{d-1}, \quad \hat{x}=x /|x|$;

$$
\Delta_{x} \rho_{n}(y, x,|k|)=\mathcal{O}\left(\frac{1}{|x|^{n+(d-1) / 2}}\right),|x| \rightarrow+\infty .
$$


We prove below the recurrent relations (25) proceeding from (135)-(139). Our approach consists in expanding equation (137) in $|x|^{-j}$, as $|x| \rightarrow+\infty$.

Note that

$$
\begin{aligned}
& \frac{\partial}{\partial r} \frac{e^{i|k| r}}{r^{\frac{d-1}{2}+j}}=\frac{e^{i|k| r}}{r^{\frac{d-1}{2}}}\left(\frac{i|k|}{r^{j}}+\frac{\left(-\frac{d-1}{2}-j\right)}{r^{j+1}}\right), \\
& \frac{\partial}{r \partial r} \frac{e^{i|k| r}}{r^{\frac{d-1}{2}+j-1}}=\frac{e^{i|k| r}}{r^{\frac{d-1}{2}}}\left(\frac{i|k|}{r^{j}}+\frac{\left(-\frac{d-1}{2}-j+1\right)}{r^{j+1}}\right), \\
& \frac{1}{r^{2}} \Delta_{S} G^{+}(x-y, k)=\frac{e^{i|k| r}}{r^{(d-1) / 2}}\left(\sum_{j=1}^{n} \frac{\Delta_{S} g_{j}(y, \hat{x},|k|)}{r^{j+1}}\right)+\mathcal{O}\left(\frac{1}{r^{n+(d+3) / 2}}\right), r \rightarrow+\infty .
\end{aligned}
$$

Using (135), (141) we obtain that

$$
\begin{aligned}
& \frac{\partial G_{n}^{+}(x-y, k)}{r \partial r}=\sum_{j=1}^{n} g_{j}(y, \hat{x},|k|) \frac{\partial}{r \partial r} \frac{e^{i|k| r}}{r^{\frac{d-1}{2}+j-1}}= \\
& =\frac{e^{i|k| r}}{r^{(d-1) / 2}} \sum_{j=1}^{n}\left(\frac{i|k| g_{j}(y, \hat{x},|k|)}{r^{j}}+\left(-\frac{d-1}{2}-j+1\right) \frac{g_{j}(y, \hat{x},|k|)}{r^{j+1}}\right)= \\
& =\frac{e^{i|k| r}}{r^{(d-1) / 2}}\left(\sum_{j=2}^{n+1} \frac{i|k| g_{j-1}(y, \hat{x},|k|)}{r^{j-1}}+\sum_{j=3}^{n+2}\left(-\frac{d-1}{2}-j+3\right) \frac{g_{j-2}(y, \hat{x},|k|)}{r^{j-1}}\right)= \\
& =\frac{e^{i|k| r}}{r^{(d-1) / 2}}\left(\frac{i|k| g_{1}(y, \hat{x},|k|)}{r}+\sum_{j=3}^{n+1} \frac{i|k| g_{j-1}(y, \hat{x},|k|)+\left(-\frac{d-1}{2}-j+3\right) g_{j-2}(y, \hat{x},|k|)}{r^{j-1}}+\right. \\
& \left.+\left(-\frac{d-1}{2}-n+1\right) \frac{g_{n}(y, \hat{x},|k|)}{r^{n+1}}\right)=\frac{e^{i|k| r}}{r^{(d-1) / 2}} \sum_{j=2}^{n} \frac{\theta_{j, d}}{r^{j-1}}, \\
& \theta_{j, d}=\left\{\begin{array}{c}
i|k| g_{j-1}(y, \hat{x},|k|), j=2, \\
i|k| g_{j-1}(y, \hat{x},|k|)+\left(-\frac{d-1}{2}-j+3\right) g_{j-2}(y, \hat{x},|k|), j=3, \ldots, n+1, \\
\left(-\frac{d-1}{2}-j+3\right) g_{j-2}(y, \hat{x},|k|), j=n+2 .
\end{array}\right.
\end{aligned}
$$

Using (141) and the result of the second equality in (143) we obtain that:

$$
\begin{aligned}
& \frac{\partial^{2} G_{n}^{+}(x-y, k)}{\partial r^{2}}=\frac{\partial}{\partial r}\left(\frac{e^{i|k| r}}{r^{(d-1) / 2}}\left(\sum_{j=1}^{n} \frac{i|k| g_{j}(y, \hat{x},|k|)}{r^{j-1}}+\sum_{j=2}^{n+1}\left(-\frac{d-1}{2}-j+2\right) \frac{g_{j-1}(y, \hat{x},|k|)}{r^{j-1}}\right)\right)= \\
& =\sum_{j=1}^{n} i|k| g_{j}(y, \hat{x},|k|) \frac{\partial}{\partial r} \frac{e^{i|k| r}}{r^{\frac{d-1}{2}+j-1}}+\sum_{j=2}^{n+1}\left(-\frac{d-1}{2}-j+2\right) g_{j-1}(y, \hat{x},|k|) \frac{\partial}{\partial r} \frac{e^{i|k| r}}{r^{\frac{d-1}{2}+j-1}}= \\
& =\sum_{j=1}^{n} i|k| g_{j}(y, \hat{x},|k|)\left(\frac{i|k| e^{i|k| r}}{r^{\frac{d-1}{2}+j-1}}+\left(-\frac{d-1}{2}-j+1\right) \frac{e^{i|k| r}}{r^{\frac{d-1}{2}+j}}\right)+ \\
& +\sum_{j=2}^{n+1}\left(-\frac{d-1}{2}-j+2\right) g_{j-1}(y, \hat{x},|k|)\left(\frac{i|k| e^{i|k| r}}{r^{\frac{d-1}{2}+j-1}}+\left(-\frac{d-1}{2}-j+1\right) \frac{e^{i|k| r}}{r^{\frac{d-1}{2}+j}}\right)=\frac{e^{i|k| r}}{r^{\frac{d-1}{2}}} \sum_{j=1}^{n} \frac{\omega_{j, d}}{r^{j-1}}, \\
& \omega_{j, d}=\left\{\begin{array}{l}
-|k|^{2} g_{j}(y, \hat{x},|k|), j=1, \\
-|k|^{2} g_{j}(y, \hat{x},|k|)+i|k|(-d-2 j+5) g_{j-1}(y, \hat{x},|k|), j=2, \\
-|k|^{2} g_{j}(y, \hat{x},|k|)+i|k|(-d-2 j+5) g_{j-1}(y, \hat{x},|k|)+ \\
+\left(-\frac{d-1}{2}-j+3\right)\left(-\frac{d-1}{2}-j+2\right) g_{j-2}(y, \hat{x},|k|), j=3, \ldots, n .
\end{array}\right.
\end{aligned}
$$


From (135), (137)-(139), (142), (143), (145) we have that

$$
\begin{aligned}
& (d-1) \frac{e^{i|k| r}}{r^{\frac{d-1}{2}}} \sum_{j=1}^{n} \frac{\theta_{j, d}}{r^{j-1}}+\frac{e^{i|k| r}}{r^{(d-1) / 2}} \sum_{j=2}^{n} \frac{\omega_{j, d}}{r^{j-1}}+\frac{e^{i|k| r}}{r^{(d-1) / 2}} \sum_{j=3}^{n+2} \frac{\Delta_{S} g_{j-2}}{r^{j-1}}= \\
& =-k^{2} \frac{e^{i|k| r}}{r^{(d-1) / 2}} \sum_{j=1}^{n} \frac{g_{j}}{r^{j-1}}+\mathcal{O}\left(\frac{|k|^{2}}{r^{n+(d-1) / 2}}\right), r \rightarrow+\infty,
\end{aligned}
$$

where $\theta_{j, d}, \omega_{j, d}, g_{j}$ do not depend on $r$. Since $n$ is arbitrary, we obtain, from (147), for $j \geq 3$, that

$$
(d-1) \theta_{j, d}+\omega_{j, d}+\Delta_{S} g_{j-2}=-k^{2} g_{j}
$$

Using definitions (144) for $\theta_{j, d},(146)$ for $\omega_{j, d}$ and equation (148) we obtain, for $j \geq 2$,

$$
g_{j}(y, \hat{x},|k|)=\frac{1}{2 i|k|(j-1)}\left(\left(\frac{4 d-3-d^{2}}{4}+(j-1)(j-2)\right) g_{j-1}(y, \hat{x},|k|)+\Delta_{S} g_{j-1}(y, \hat{x},|k|)\right) .
$$

Formulas (25) follow from (136), (149).

\section{Acknowledgments}

V N Sivkin was supported by Theoretical Physics and Mathematics Advancement Foundation "BASIS", Grant № 20-8-10-5-1.

\section{References}

1. A.D. Agaltsov, T. Hohage, R.G. Novikov, An iterative approach to monochromatic phaseless inverse scattering, Inverse Problems 35(2), 24001 (24 pp.) (2019)

2. T. Aktosun, P. E. Sacks, Inverse problem on the line without phase information, Inverse Problems 14, 211-224 (1998)

3. F. V. Atkinson, On Sommerfeld's "Radiation Condition", Philos. Mag., Vol. XI, 645-651 (1949)

4. A.H. Barnett, Ch.L. Epstein, L.F. Greengard, J.F. Magland, Geometry of the phase retrieval problem, Inverse Problems 36, 094003 (2020)

5. F.A. Berezin, M.A. Shubin, The Schrödinger Equation, Mathematics and Its Applications, Vol. 66, Kluwer Academic, Dordrecht, 1991

6. M. Born, Quantenmechanik der Stossvorgange, Zeitschrift fur Physik 38 (11-12), 803-827 (1926)

7. V.A. Burov, O.D. Rumyantseva, Inverse wave problems of acoustic tomography, Part 2 : Inverse problems of acoustic scattering, Lenand, Moscow, (in Russian) 2020

8. K. Chadan, P.C. Sabatier, Inverse Problems in Quantum Scattering Theory, 2nd edn. Springer, Berlin, 1989

9. D. Colton, R. Kress, Inverse Acoustic and Electromagnetic Scattering Theory, 4th. ed. Applied Mathematical Sciences, 93. Springer, Cham, 2019

10. L. Crocco, M. D'Urso, T. Isernia, Inverse scattering from phaseless measurements of the total field on a closed curve, Journal of the Optical Society of America A 21(4), 622-631 (2004)

11. A.J. Devaney, Structure determination from intensity measurements in scattering experiments, Physical Review Letters 62(20), 2385-2388 (1989)

12. L.D. Faddeev, S.P. Merkuriev, Quantum Scattering Theory for Multi-particle Systems, Mathematical Physics and Applied Mathematics, 11. Kluwer Academic Publishers Group, Dordrecht, 1993 
13. D. Fanelli, O. Öktem, Electron tomography: a short overview with an emphasis on the absorption potential model for the forward problem, Inverse Problems, 24(1), 013001 (51 pp.) (2008)

14. A. Jesacher, W. Harm, S. Bernet, M. Ritsch-Marte, Quantitative single-shot imaging of complex objects using phase retrieval with a designed periphery, Optics Express 20(5), 5470-5480 (2012)

15. O. Ivanyshyn, R. Kress, Identification of sound-soft 3D obstacles from phaseless data, Inverse Probl. Imaging 4, 131-149 (2010)

16. P. Jonas, A.K. Louis, Phase contrast tomography using holographic measurements, Inverse Problems 20(1), 75-102 (2004)

17. T. Hohage, R.G. Novikov, Inverse wave propagation problems without phase information, Inverse Problems 35(7), 070301 (4 pp.)(2019)

18. M.V. Klibanov, Phaseless inverse scattering problems in three dimensions, SIAM J.Appl. Math. 74(2), 392-410 (2014)

19. M.V. Klibanov, N.A. Koshev, D.-L. Nguyen, L.H. Nguyen, A. Brettin, V.N. Astratov, A numerical method to solve a phaseless coefficient inverse problem from a single measurement of experimental data, SIAM J. Imaging Sci. 11(4), 2339-2367 (2018)

20. M.V. Klibanov, V.G. Romanov, Reconstruction procedures for two inverse scattering problems without the phase information, SIAM J. Appl. Math. 76(1), 178-196 (2016)

21. M.V. Klibanov, P.E. Sacks, A.V. Tikhonravov, The phase retrieval problem, Inverse Problems 11, 1-28 (1995)

22. M.H. Maleki, A.J. Devaney, A. Schatzberg, Tomographic reconstruction from optical scattered intensities, Journal of the Optical Society of America A 9(8), 1356-1363 (1992)

23. S. Maretzke, A uniqueness result for propagation-based phase contrast imaging from a single measurement, Inverse Problems 31(6), 065003 (16 pp.) (2015)

24. S. Maretzke, T. Hohage, Stability estimates for linearized near-field phase retrieval in X-ray phase contrast imaging, SIAM J. Appl. Math. 77(2), 384-408 (2017)

25. R.B. Melrose, Geometric scattering theory, Stanford Lectures. Cambridge University Press, 1995

26. R. G. Novikov, Formulas for phase recovering from phaseless scattering data at fixed frequency, Bulletin des Sciences Mathematiques 139(8), 923-936 (2015)

27. R. G. Novikov, Phaseless inverse scattering in the one-dimensional case, Eurasian Journal of Mathematical and Computer Applications 3(1), 63-69 (2015)

28. R. G. Novikov, Inverse scattering without phase information, Seminaire Laurent Schwartz - EDP et applications, Exp. No16, (13pp) (2014-2015)

29. R. G. Novikov, Multipoint formulas for phase recovering from phaseless scattering data, Journal of Geometric Analysis 31(2), 1965-1991 (2021)

30. R.G. Novikov, Multidimensional inverse scattering for the Schrödinger equation, Book series: Springer Proceedings in Mathematics and Statistics. Title of volume: Mathematical Analysis, its Applications and Computation - ISAAC 2019, Aveiro, Portugal, July 29-August 2; Editors: P. Cerejeiras, M. Reissig (to appear), e-preprint: https://hal.archives-ouvertes.fr/hal-02465839v1

31. R. G. Novikov, Multipoint formulas for scattered far field in multidimensions, Inverse Problems 36(9), 095001 (12 pp) (2020)

32. R.G. Novikov, Multipoint formulas for inverse scattering at high energies, Russian Math. Surveys (to appear), https:// hal.archives-ouvertes.fr/hal-02983682v1

33. R. G. Novikov, V. N. Sivkin, Error estimates for phase recovering from phaseless scattering data, Eurasian Journal of Mathematical and Computer Applications, 8(1), 44-61 (2020)

34. R. G. Novikov, V. N. Sivkin, Phaseless inverse scattering with background information, Inverse Problems, vol. $37(5), 055011$ (20 pp) (2021) 
35. V.Palamodov, A fast method of reconstruction for X-ray phase contrast imaging with arbitrary Fresnel number, arXiv:1803.08938v1 (2018)

36. V. G. Romanov, Inverse problems without phase information that use wave interference, Sib. Math. J. 59(3), 494-504 (2018)

37. V. G. Romanov, Phaseless inverse problems for Schrödinger, Helmholtz, and Maxwell equations, Comput. Math. Math. Phys., 60 (6) 1045-1062 (2020)

38. V.G. Romanov, A phaseless inverse problem for electrodynamic equations in the dispersible medium, Applicable Analysis, https://doi.org/10.1080/00036811.2020.1846721

39. C. H. Wilcox, A generalization of theorems of Rellich and Atkinson, Proceedings of the American Mathematical Society, 7(2), 271-276 (1956)

40. E. Wolf, Three-dimensional structure determination of semi-transparent objects from holographic data, Optics Communications 1(4), 153-156 (1969)

41. E. Wolf, Determination of the amplitude and the phase of scattered fields by holography, Journal of the Optical Society of America 60(1), 18-20 (1970)

42. X. Xu, B. Zhang, H. Zhang, Uniqueness in inverse electromagnetic scattering problem with phaseless far-field data at a fixed frequency, IMA Journal of Applied Mathematics 85(6), 823-839 (2020) 